\title{
Socioeconomic Status, Sex, Age, and Ethnicity as Determinants of Body Fat Distribution for Guatemalan Children
}

\author{
BARRY BOGIN AND TIMOTHY SULLIVAN \\ Department of Behavioral Sciences, University of Michigan, \\ Dearborn, Michigan 48128
}

\begin{abstract}
KEY WORDS Fat distribution, Fatness, SES, Sexual dimorphism,
\end{abstract} Age, Ethnicity, Guatemala

\begin{abstract}
The distribution of subcutaneous fat at the triceps and subscapular skinfold sites is described for four groups of children living in Guatemala. These groups are (1) high socioeconomic status (SES) children of Ladino (mixed Spanish and Indian) ancestry, (2) high SES children of European ancestry, (3) low SES Ladino children, and (4) very low SES Indian children. The method of Healy and Tanner (1981) is used, employing regression and principal components analysis of log transformed skinfold values to divide "fatness" into two uncorrelated variables: size (amount of fat) and shape (fat pattern). Significant differences exist between groups in size, with lower SES groups having less fat than higher SES groups. No significant difference in fat pattern exists between the high SES Ladino and high SES European children. Significant differences do exist between the high SES groups and the low SES groups. The relative amount of subscapular fat increases from the high SES Ladinos and high SES Europeans, to the low SES Ladinos, to the very low SES Indians. In the high SES European and high SES Ladino samples, girls have significantly more arm fat than boys. There is no significant difference in fat patterning between boys and girls in the two low SES samples. Finally, the relative amount of subscapular fat tends to increase with age in all four samples. These results indicate that (1) fatness and fat patterning are independent anatomical characteristics, (2) SES influences fat patterning; low SES children of both Ladino and Indian ancestry show greater reductions in arm fat than in trunk fat compared to high SES children, (3) sexual dimorphism in fat patterning is SES dependent; low SES children show no dimorphism, high SES children are dimorphic, (4) ethnic differences in fat patterning are not demonstrable for Ladinos and Europeans of high SES; fat distribution differences between low SES Ladinos and very low SES Indians may be due to ethnic factors, SES differences, or both.
\end{abstract}

The anatomical distribution of subcutaneous fat on the human body is not uniform. Rather, different sites of the body accumulate unequal amounts of the total fat store. Population or ethnic differences in fat distribution are well known. The causes of these differences are not understood. Since subcutaneous fat distribution is significantly associated with several diseases such as diabetes, coronary heart disease, and hypertension (Vague, 1956; Hunt, 1972; Kissebah et al., 1982; Mueller et al., 1984), the ethnic differ- ences are of practical as well as theoretical importance.

As an example of the practical importance of the ethnic differences in fat distribution, we cite a study by Johnston et al. (1984). That study compares the international reference data of the U.S. Center for Health Statistics (NCHS) with local reference data from Guatemala for the triceps and subscapular skin.

Received May 9, 1985; revision accepted October 29, 1985. 
fold. The Guatemalan sample is of children and youth of high socioeconomic status (SES) and free of the nutritional and health insults that adversely affect the growth of much of the lower SES segments of Guatemalan society. It is found that, in general, Guatemalan children and youth have less fat on their arms and more fat on their trunks than do age-matched subjects of the NCHS references. Figures 1 and 2 illustrate the populational differences in fat distribution for girls. The practical significance of this finding is that local reference data may be more appropriate to use when assessing body composition, nutritional status, and anthropometric risk factors for chronic diseases.

It has been reported that environmental factors such as diet and exercise have little or no influence on individual or populational differences in fat patterning (Garn, 1955). For this reason it is often assumed that these differences have a major genetic component (Malina, 1966; Robson et al., 1971; West, 1978; Mueller and Reid, 1979; Malina et al., 1982). Those researchers who allow for some environmental effect state that the degree to which environmental factors influence fat patterning is unknown (Malina et al., 1982; Mueller, 1982; Mueller et al., 1982; Deutsch, et al., 1985).

Virtually all the populational analyses of fat patterning carried out so far have compared samples from different geographical regions (e.g., Asians versus Europeans), from different sociocultural environments within the same region (e.g., blacks and whites from the United States), or both. Thus, the widespread belief that ethnic differences in fat pattern are primarily genetically determined cannot be accepted at face value. The effect of local environments, both physical and cultural, may also influence fat patterning.

In this paper we analyze the patterns of fat distribution of boys and girls of different ethnic and socioeconomic status living in Guatemala. We show that for these children, environmental influences associated with SES are possibly more important than ethnicity in determining subcutaneous fat distribution.

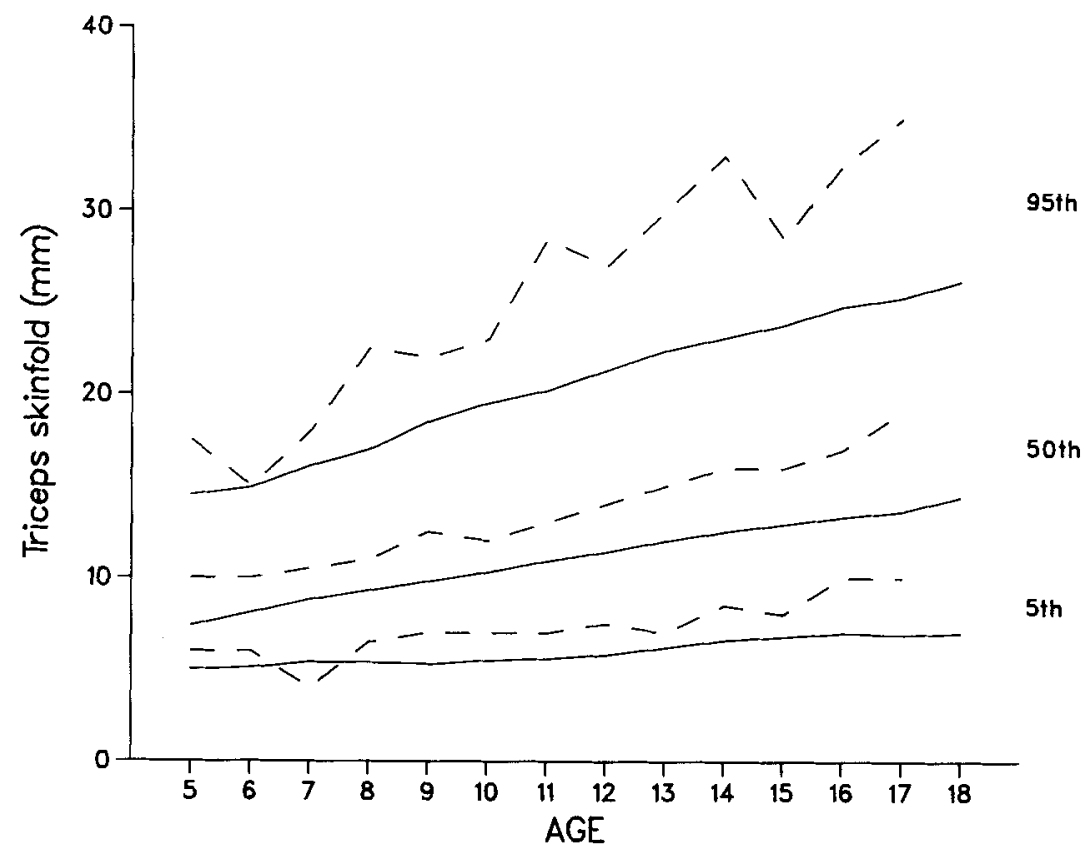

Fig. 1. Comparison of the 95th, 50th, and 5th percentiles of the triceps skinfold for female subjects of the United States NCHS standards (dashed lines) and the Guatemalan reference data (solid lines). [From Johnston et al., 1984.] 


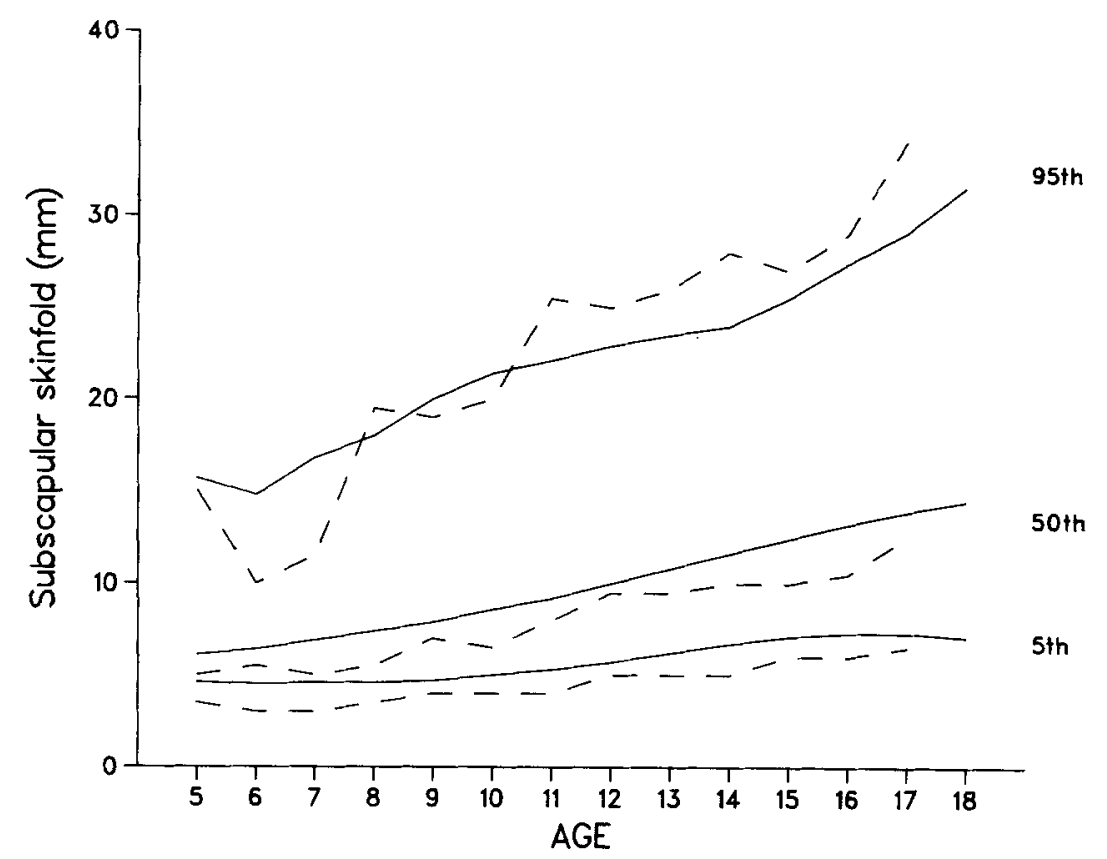

Fig. 2. Comparison of the 95th, 50th, and 5th percentiles of the subscapular skinfold for female subjects of the United States NCHS standards (dashed lines) and the Guatemalan reference data (solid lines). [From Johnston et al., 1984.]

\section{MATERIALS AND METHODS}

The data are derived from the Longitudinal Study of Urban Child Development conducted by the Universidad del Valle de Guatemala. The sample of this study includes children of different SES and ethnic statuses, attending school in and near Guatemala City. For the present analysis we selected four groups of children. These are (1) high SES children of European ancestry (81 boys and 83 girls), (2) high SES Ladino children (177 boys and 143 girls), (3) low SES Ladino children (188 boys and 152 girls), and (4) very low SES Cakchiquel Indian children (395 boys and 274 girls). All children are between the ages of 7.00 and 13.99 years. Europeans are those children who have parents and grandparents born in, mostly, northwestern and central Europe or in North America and who have non-Hispanic surnames. Ladinos are Guatemalans of mixed Spanish and Indian ancestry who speak Spanish and have a generally "Western" set of cultural values. In our samples, Ladinos were further identified as having both parents and all four grandparents born in Guatemala and having Spanish surnames. Indians are the children of native Guatemalans from the Cakchiquel language and culture group. They speak Cakchiquel as a first language, dress in nonwestern clothing, and follow Indian religious practices.

The high SES Europeans and high SES Ladinos attend the same private school in Guatemala City. Owing to their privileged home and school environments, these children are free of major health and nutritional problems, and their growth closely follows the pattern of growth of healthy children from the developed nations, such as the United States (Johnston et al., 1973). The low SES Ladino children attend a government sponsored public school in Guatemala City. These children have been shown to suffer from mild-to-moderate energy malnutrition and experience growth delays compared to high SES Ladinos (Bogin and MacVean, 1978, 1981a). The Indian children attend the gov- 
ernment operated public school in a semirural village 22 kilometers from Guatemala City. The village consists of virtually all Cakchiquel Indians. The economy of the village is based upon textile and clothing manufacture, rather than traditional agriculture. Though the villagers are part of a cash economy, poverty is widespread in the village. Compared with high SES Ladino children, the growth of the Indian children is even more delayed than that of low SES Ladinos. Indeed, the growth of these nonagrarian Indian children is very similar to that of traditional agrarian Indians living in rural villages in Mexico and Guatemala (Bogin and MacVean, 1984).

Triceps and subscapular skinfolds were measured for each child. Duplicate measurements were taken by trained assistants employing Lange calipers. Details of measurement procedures, reliabilities, and the original measurements are given in previous publications (Bogin and MacVean, 1981a, 1984).

In this paper we consider fat distribution, the relative amount of subcutaneous fat at the triceps versus the subscapular skinfold sites. We use the method of Healy and Tanner (1981) for the analysis of size and shape in relation to growth status. The method involves three steps: (1) transformation of each skinfold measurement to its logarithm-size is then computed as the unweighted mean of all the logarithms; (2) regression of each log skinfold on the mean log of all skinfoldsshape is then computed as the residual values from the regressions; (3) the residuals are then subjected to principal components analysis, and principal component scores are computed; these scores represent an index of fat distribution, in this case the relative degree of trunk versus extremity fatness.

The advantages of this method are that it allows for the description of fat distribution differences between individuals or groups independent of their degree of total fatness, since the measure of shape is described as everything residual to size. The method also avoids problems related to units of measurement and differences in the coefficients of variation for each variable. The latter is a problem inherent in the use of simple ratios and Z-score transformations (Tanner, 1951). Finally, most growth variables are related to each other in nonlinear ways. For instance, increases in total fatness may increase the size of the triceps skinfold absolutely more than the size of the subscapular skinfold. The Healy and Tanner method converts nonlinear allometric relations between growth variables to a linear expression, e.g., before

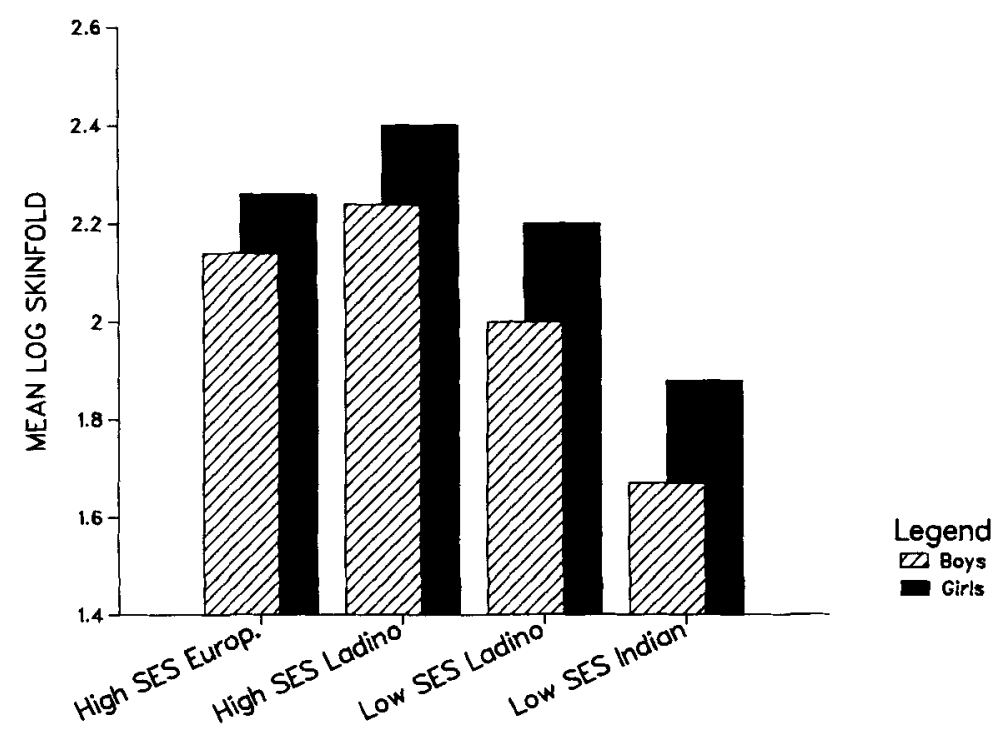

Fig. 3. Mean log skinfold values (fatness) for boys and girls of each sample. Age effects were removed by analysis of covariance. Exact age adjusted mean values $(+/$ - SE) are high SES boys $=2.14(.04)$, girls $=2.27(.04)$;

high SES Ladino boys $=2.24(.03)$, girls $=2.40(.03)$; low SES Ladino boys $=2.00(.03)$, girls $=2.20(.03)$; very low SES Indian boys $=1.67(.02)$, girls $=1.88(.02)$. 
transformation, $\mathrm{y}=\mathrm{aX}{ }^{\mathrm{b}}$; after transformation, $\mathrm{y}=\ln (\mathrm{a})+\mathrm{b}(\mathrm{1n})(\mathrm{X})$. This allows the use of linear statistical models for analysis.

In all the subsequent analyses, a probability level of .05 or less is employed to determine statistical significance.

\section{RESULTS}

Figure 3 summarizes the degree of fatness achieved by boys and girls in each sample. The data are for the means of the sum of the $\log$ transformations of the subscapular and triceps skinfolds. The effect of age (the children are between 7 and 13 years old) was statistically removed by analysis of covariance on the log transformed data. Girls are significantly fatter than boys in each sample. High SES Ladino boys and girls are significantly fatter than their high SES European peers. High SES Ladino and high SES European boys are significantly fatter than low SES Ladino or Indian boys. High SES Ladino girls are significantly fatter than Indian girls; however, they are not statistically fatter than low SES Ladino girls. Note also the relative leanness of the Indian boys and girls compared with all other groups.

TABLE 1. Principal components (PC) analysis of residual log skinfold measurements

\begin{tabular}{|c|c|c|}
\hline & PC 1 & $\mathrm{PC} 2$ \\
\hline Eigenvalue & 1.75 & 0.25 \\
\hline Percent variance & 87.36 & 12.64 \\
\hline \multicolumn{3}{|l|}{ Eigenvectors } \\
\hline $\begin{array}{l}\text { Residual log } \\
\text { triceps }\end{array}$ & +0.71 & 0.71 \\
\hline $\begin{array}{l}\text { Residual log } \\
\text { subscapular }\end{array}$ & -0.71 & 0.71 \\
\hline
\end{tabular}

Fat distribution differences between samples were evaluated by the principal components analysis. The principal components are given in Table 1. Results for boys and girls were computed separately, but were identical. The first component, which accounts for $87 \%$ of the variance, contrasts the two skinfolds. Triceps loads positively and subscapular loads negatively on this measure of shape (fat distribution). The skinfolds load equally on the second component, which is the measure of size (fatness). The second component is not significant; its eigenvalue is less than 1.00 , thus demonstrating the statistical independence of shape and size from this method of analysis.

A multiple regression analysis was employed to evaluate the influence of group, sex, and age on the principal component scores. For the regression, the principle component scores are the dependent variable. Sample, age, and sex are treated as categorical independent variables. The results are presented in Table 2. Each of the three independent variables has a significant effect on fat distribution. However, the total variance accounted for by these variables is relatively small. The variable "sample" has the largest partial correlation and beta weight with shape. The sample effect may be due to either ethnic differences (European, Ladino, Indian), to SES differences (high, low, very low), or to the interaction of both.

Table 3 lists the descriptive statistics for the fat distribution scores of boys from each of the four samples by age. Similar statistics for the girls may be found in Table 4. Larger score values indicate relatively more fat at the triceps site rather than the subscapular site. The general trend in each sample is for

TABLE 2. Regression of principal component score (fat distribution) on the categorical independent variables age, sex, and sample ${ }^{1}$

\begin{tabular}{lccccr}
\hline $\begin{array}{l}\text { Source of } \\
\text { variation }\end{array}$ & DF & $\begin{array}{c}\text { Sum } \\
\text { squares }\end{array}$ & $\begin{array}{c}\text { Mean } \\
\text { square }\end{array}$ & F-statistic & P \\
\hline $\begin{array}{l}\text { Regression } \\
\text { Error }\end{array}$ & 1,646 & .12 & .04 & 71.49 & $<.00$ \\
\hline Variable & Partial & $\begin{array}{c}\text { Beta } \\
\text { weight }\end{array}$ & $\begin{array}{c}\text { Standard } \\
\text { error }\end{array}$ & T-statistic & $\begin{array}{r}\text { Signi- } \\
\text { ficance }\end{array}$ \\
\hline Age & & .20 & .001 & 8.33 & $<.00$ \\
Sex & -.20 & .05 & .02 & 2.00 & .05 \\
Sample & .05 & .31 & .02 & 13.07 & $<.00$ \\
\hline
\end{tabular}

${ }^{1}$ Mult $\mathrm{R}=.34 ; \mathrm{R}-\mathrm{Sqr}=.12 ; \mathrm{SE}=.02$. 

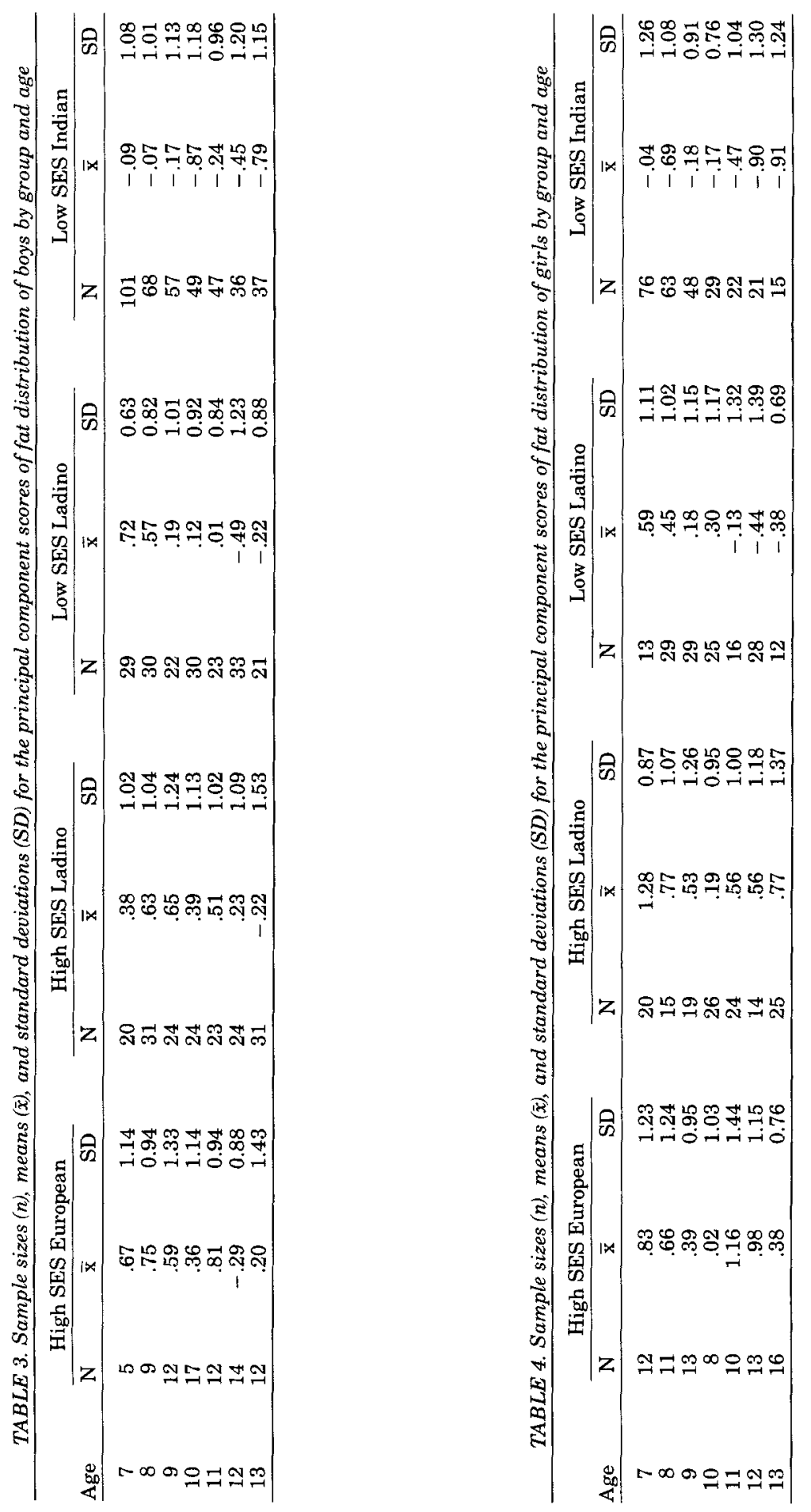
a decrease in score values with age. However, small sample sizes for many of the ages results in erratic trends for some groups. Despite this limitation, it may be noted that the tendency for subcutaneous fat to become more centripetally located with age is commonly found in many populations (Mueller, 1982). It is important to note the negative scores for the Indian boys and girls at all ages and for the low SES Ladino boys and girls at older ages. These negative scores indicate a pronounced concentration of fat on the trunk of the body rather than on the extremities.

Owing to sample size limitations at some ages, further analysis of the age effects on fat distribution may not be reliable. Therefore, for the analysis of group and sex effects, age was statistically removed as a variable by analysis of covariance.

The effects of group and sex on fat distribution are presented in Figure 4 . The data are for the age-adjusted mean principal component scores. There is no significant difference in the scores between the high SES European and high SES Ladino boys. The scores of the low SES Ladino and Indian boys are significantly smaller than those of the high SES boys $(P<.05)$. In turn, Indian boys have a score significantly smaller than that of the low SES Ladino boys $(\mathrm{P}<.05)$. There is no difference in the scores between the two groups of high SES girls. Low SES Ladino girls have a significantly smaller ratio than the two groups of high SES girls. Low SES Indian girls have a significantly smaller ratio than low SES Ladino girls $(P<.05)$. Thus, relative trunk fatness of the boys and the girls increases as socioeconomic status decreases.

Figure 4 also shows an interaction between SES and sex on fat distribution scores. For the two high SES samples, the girls have significantly larger scores than the boys $(\mathrm{P}$ $<.05$ ). This means that these well nourished girls have relatively more arm fat while their male counterparts have relatively more trunk fat. This pattern is reversed in the two low SES samples. Boys in these less well nourished samples have relatively more arm fat than the girls. However, the difference is not statistically significant. Thus, the interaction between sex and SES is that only the high SES samples show a significant difference in fat patterning between the sexes.

\section{DISCUSSION}

The results of this analysis of fat distribution in children of different ethnic and socioeconomic backgrounds demonstrates four main points. First, fatness and fat patterning are independent aspects of body composition.

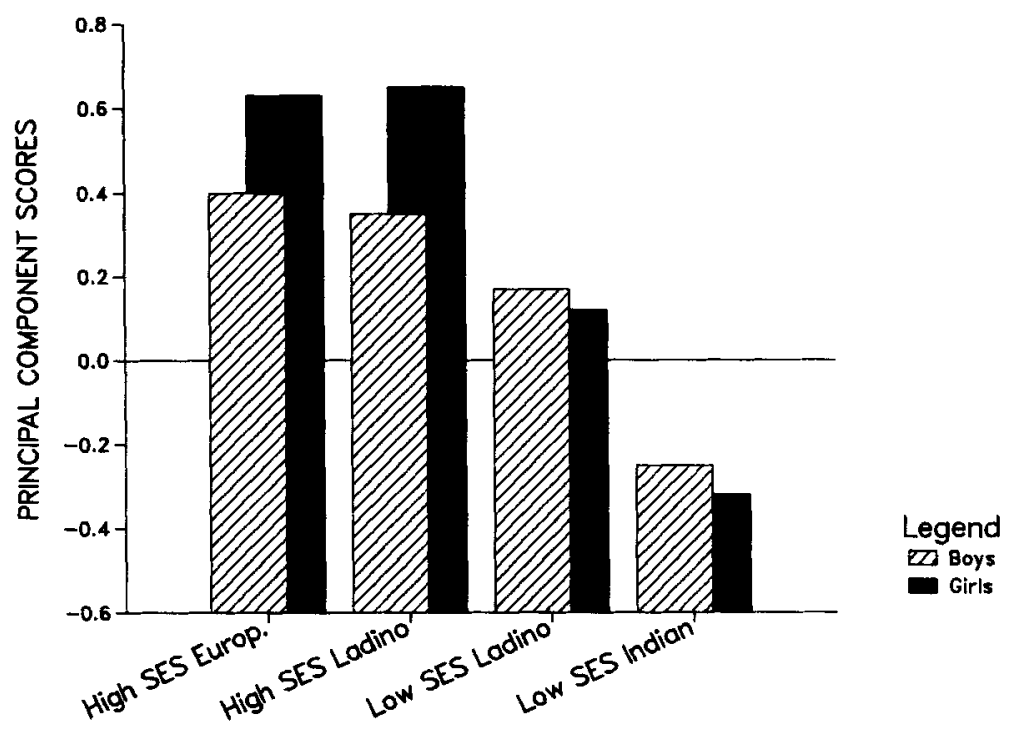

Fig. 4. Principal component scores (fat distribution) for boys and girls of each sample. Age effects were removed by analysis of covariance. Exact age-adjusted mean values $(+/-$ SE) are high SES boys $=.40(.13)$, girls $=.63$
(.13); high SES Ladino boys $=.35(.07)$, girls $=.65(.10)$; Iow SES Ladino boys $=.17(.08)$, girls $=.12(.09)$; very low SES Indian boys $=-.25$ (.06), girls $=-.32(.07)$. 
This point has been made by other studies as well (Mueller and Reid, 1979). Second, socioeconomic status influences fat stores distribution. As SES decreases, subcutaneous fat become more centripetal, that is, relative amounts of arm fat decrease and relative amounts of trunk fat increase. Third, sexual dimorphism in fat distribution is SES dependent. Low SES children are not dimorphic, whereas high SES children are dimorphic, with boys showing a more centripetal fat pattern than girls. Fourth, ethnic differences in fat distribution are not easily demonstrable. There are no differences between high SES European and Ladino children. One would predict that any genetic potential for fat pattern differences would most likely be expressed between these two well fed and healthy ethnic groups. Indeed, these two populations are known to have significant genetic differences at the ABO locus (Johnston et al., 1973). Low SES Ladino and Indian children differ in fat distribution. Unfortunately, it is not possible to attribute this difference to their ethnicity or to the lower SES, and its sequelae for growth and development, of the Indian group.

As was shown in Figures 1 and 2. significant differences in fatness at the triceps and subscapular skinfold sites exist between high SES Guatemalans living in Guatemala and children of predominantly European origin living in the United States (Johnston et al., 1984). Our present finding of no such difference in fat distribution between high SES Ladino and European children living in Guatemala apparently indicates that factors common to the overall environment of these two groups of children, for example, diet and activity regimes, are the major determinants of their body composition. Our analysis, using principal components to define fat patterns, supports an earlier finding of no significant differences in fatness at the triceps or subscapular skinfold sites between high SES European and high SES Ladino children in Guatemala (Johnston et al., 1975). Our study and the earlier one are based on independent samples from these two populations. In combination, these studies show that there is no statistically discernible ethnic contribution to subcutaneous fat distribution for these two populations of children.

An environmental model for fat pattern determination may be extended to include the findings for the low SES Ladino and very low SES Indian samples. Each of these samples shows a significant increase in centripe- tal fat distribution compared with the high SES samples. Previous publications show that the low SES Ladino and Indian children suffer from mild-to-moderate energy malnutrition, and that the latter suffer to a greater degree than the former. It is also known that under nutritional stress these poor Guatemalan children experience greater reductions in fat stored at the triceps site than at the subscapular site (Bogin and MacVean, 1981a,b; 1984). The present paper supports the conclusion that changes in fat patterning occur with energy malnutrition. Thus, socioeconomic status, a proxy measure for nutritional adequacy and other environmental variables, correlates with fat distribution across the entire range of ethnic groups considered in this analysis.

Other researchers investigating the determinants of fat patterning leave open the question of the importance of environmental influences. Deutsch et al. (1985) point out that, ". . little is known about the development of this characteristic [fat patterning] or about its genetic and environmental determinants." Mueller (1982) suggests that the well-known black-white differences in fat patterning could be partly environmentally determined. Ramirez and Mueller (1980) find that fat patterning becomes more centripetal in Polynesians migrating from traditional rural to modern urban environments. Acculturation to Western society and "modernization" are associated with increased fatness, an increase in centralized (trunk) obesity, and increased rates of maturity-onset diabetes among Dogrib Indians in Canada (Szathmary and Holt, 1983) and Mexican-Americans in Texas (Mueller et al., 1984). Similarly, migration from the islands of Samoa to Hawaii and California and acculturation to Western society are associated with increased fatness, an increase in centralized obesity, hypertension, and greater risk for diabetes in Samoans (Pawson and Janes, 1981; McGarvey, 1984; Bindon and Baker, 1985).

These studies, plus our present findings, leave little doubt that environmental factors can alter fat patterning. It seems that we must now demonstrate to what extent genetic factors influence fat distribution. Such evidence will be difficult to acquire owing to the multiple and subtle ways in which cultural, social, and geographical differences between human populations influence human biology. However, given the significant asso- 
ciations between fat patterning and several diseases of major public health importance (diabetes, atherosclerosis, hypertension, cancer, etc.), it is essential that we know how environment and genetics influence the deposition of subcutaneous fat on the human body.

\section{ACKNOWLEDGMENTS}

Dr. William $\mathrm{H}$. Mueller suggested the statistical method used in this paper and provided helpful criticism of an earlier draft. Dr. Michael LaChance helped clarify some of the mathematical difficulties of our problem. Dr. William Stini and anonymous reviewers provided many suggestions for improvement. We thank them all.

\section{LITERATURE CITED}

Bindon, JR, and Baker, PT (1985) Modernization, migration and obesity among Samoan adults. Ann. Hum. Biol. 12:67-76.

Bogin, B, and MacVean, RB (1978) Growth in height and weight of urban Guatemalan primary school children of low and high socioeconomic class. Hum. Biol. 50:477 487.

Bogin, B, and MacVean, RB (1981a) Body composition and nutritional status of urban Guatemalan children of high and low socioeconomic class. Am. J. Phys. Anthropol. 55:543-551.

Bogin, B, and MacVean, RB (1981b) Nutritional and biological determinants of body fat patterning in urban Guatemalan children. Hum. Biol. 53:259-268.

Bogin, B, and MacVean, RB (1984) Growth status of nonrarian, semi-urban living Indians in Guatemala. Hum. Biol. 56:527-538.

Deutsch, MI, Mueller, WH, and Malina, RM (1985) An drogyny in fat patterning is associated with obesity in adolescents and young adults. Ann. Hum. Biol. 12:275286.

Garn, SM (1955) Relative fat patterning, an individual characteristic. Hum. Biol. 27:75-89.

Healy, MJR, and Tanner, JM (1981) Size and shape in relation to growth and form. Symp. Zool. Soc. Lond. 46:19-35

Hunt, EE (1972) Obesity: Epidemiological considerations. Adv. Psychosom. Med. 7:148-163.

Johnston, FE, Bogin, B, MacVean, RB, and Newman, BC (1984) A comparison of international standards versus local reference data for the triceps and subscapular skinfolds of Guatemalan children and youth. Hum. Biol. 56:157-171.
Johnston, FE, Borden, M, and MacVean, RB (1973) Height, weight and their growth velocities in Guatemalan private school children of high socio-economic class. Hum. Biol. 45:627-641.

Johnston, FE, Dechow, PC, and MacVean, RB (1975) Age changes in skinfold thickness among upper class school children of differing ethnic backgrounds residing in Guatemala. Hum. Biol. 47:251-262.

Kissebah, AH, Vydelingum, N, Murry, R, Evans, DJ, Hartz, AJ, Kalkhoff, RK, and Adams, PW (1982) Relation of body fat distribution to metabolic complications of obesity. J. Clin. Endocrinol. Metab. 54:254-260.

Malina, RM (1966) Patterns of development in skinfolds of Negro and white Philadelphia children. Hum. Biol. 38:89-103.

Malina, RM, Mueller, WH, Bouchard, C, Shoup, RF, and Lariviere, G (1982) Fatness and fat patterning among athletes at the Montreal Olympic games. Med. Sci. Sports Exerc. 14:445-452

McGarvey, ST (1984) Subcutaneous fat distribution and blood pressure of Samoans. (Abstract) Am. J. Phys. Anthropol. 63:192.

Mueller, WH (1982) The changes with age of the anatomical distribution of fat. Soc. Sci. Med. 16:191-196.

Mueller, WH, Joos, SK, Hanis, CL, Zavalita, AN, Eichner, J and Schull, WJ (1984) Diabetes alert study: Growth, fatness and fat patterning, adolescence through adulthood, in Mexican-Americans. Am. J. Phys. Anthropol., 64:389-399.

Mueller, WH, and Reid, RM (1979) A multivariate analysis of fatness and relative fat patterning. Am. J. Phys. Anthropol. 50:199-208.

Mueller, WH, Shoup, RF, and Malina, RM (1982) Fat patterning in athletes in relation to ethnic origin and sport. Ann. Hum. Biol. 9:371-376.

Pawson, IG, and Janes, C (1981) Massive obesity in a migrant Samoan population. Am. J. Pub. Health 71:508-513.

Ramirez, ME, and Mueller, WH (1980) The development of obesity and fat patterning in Tokelau children. Hum. Biol. 52:675-688.

Robson, JRK, Bazin, M, and Soderstrom, R (1971) Ethnic differences in skinfold thickness. Am. J. Clin. Nutr. 24:864-868

Szathmary, EJE, and Holt, N (1983) Hypoglycemia in Dogrib Indians of the Northwest Territories, Canada: Association with age and a centripetal distribution of body fat. Hum. Biol. 55:493-515.

Tanner, JM (1951) Some notes on the reporting of growth data. Hum. Biol. 23:9-159.

Vague, J (1956) The degree of masculine differentiation of obesities: A factor determining predisposition to diabetes, atherosclerosis, gout and uric calculous dis ease. Am. J. Clin. Nutr. 4:20-34

West, KM (1978) Diabetes in American Indians. Adv. Metab. Disord. 9:29-48. 